Int. J. Morphol.,

32(3):991-997, 2014

\title{
Resultados Iniciales de la Cirugía de la Obesidad con Gastrectomía Vertical y By-Pass de Yeyuno
}

\author{
Initial Results of Weight Loss Surgery with Vertical Gastrectomy and Jejunal By-pass
}

\author{
Carlos Manterola ${ }^{* * *, * * *}$; Munir Alamo****; Jaime Horta**; Miguel Ángel Icarte**; \\ Claudia Riveros**; Carlos Ayala** \& Estela Mendoza**
}

\begin{abstract}
MANTEROLA, C.; ALAMO, M.; HORTA, J.; ICARTE, M. A.; RIVEROS, C.; AYALA, C. \& MENDOZA, E. Resultados iniciales de la cirugía de la obesidad con gastrectomía vertical y by-pass de yeyuno. Int. J. Morphol., 32(3):991-997, 2014.

RESUMEN: La obesidad mórbida (OM), es una enfermedad crónica que se asocia a riesgo de morbilidad y mortalidad. La cirugía ha demostrado ser la mejor opción terapéutica. El objetivo de este estudio, fue reportar los resultados de una serie de pacientes intervenidos quirúrgicamente por OM con gastrectomía vertical y by-pass de yeyuno (GVBY), en términos de reducción del IMC. Serie de casos prospectiva. Se incluyeron todos los pacientes intervenidos quirúrgicamente por OM, en el Servicio de Cirugía del Hospital Clínico de la Universidad Mayor, Temuco, Chile; en el período 2009 y 2014. Todos los pacientes fueron operados con una GVBY. La variable resultado fue reducción del IMC. Otras variables de interés fueron tiempo quirúrgico, estancia hospitalaria, morbilidad postoperatoria (MPO), pérdida del exceso de peso (PEP), porcentaje de la pérdida del exceso de peso (\%PEP), reducción de comorbilidad asociada y mortalidad. Se utilizó estadística descriptiva con cálculo de porcentajes, medidas de tendencia central y dispersión. Se intervinieron 30 pacientes, con una mediana de edad de 38 años; con un 73,3\% de sexo femenino. Se verificó una reducción progresiva y sostenida del peso, del IMC (normalizado en promedio a los 12 meses), de la PEP y del \% PEP a lo largo del tiempo. La reducción general de co-morbilidad fue de $100 \%$ y la resolución total de la comorbilidad general fue de $91,1 \%$. La mediana del tiempo quirúrgico y de la estancia hospitalaria fue de 125 min y 3 días respectivamente. La MPO fue de 13,3\% (dos casos de fuga anastomótica y dos de hemorragia digestiva alta). La serie no reporta mortalidad. Los resultados observados con GVBY son comparables con los de otras series de cirugía de OM.
\end{abstract}

PALABRAS CLAVE: Obesidad mórbida; Cirugía bariátrica; Cirugía de control metabólico; Índice de masa corporal.

\section{INTRODUCCIÓN}

La obesidad mórbida (OM), es una enfermedad crónica que se asocia con un mayor riesgo de morbilidad y mortalidad. Su prevalencia es creciente en el mundo occidental ( $20 \%$ en el Reino Unido y 22,0\% en EE.UU.), con importantes consecuencias en la calidad y expectativa de vida de los pacientes. En Chile, en la Región Metropolitana, la prevalencia es de $35,9 \%$ de obesidad en población de mayores de 15 años de edad; estimándose que existen 4 millones de personas obesas en el país. Por otra parte, estudios del Ministerio de Salud demuestran que representa la segunda causa de años de vida perdidos por muerte o por discapacidad prematura y la sexta causa de muerte a nivel nacional (Atalah, 2014).

El obeso con un índice de masa corporal (IMC) superior a $40 \mathrm{Kg} / \mathrm{m}^{2}$ es víctima de una enfermedad conocida como OM y la cirugía bariátrica (CB) en conjunto con un estricto apoyo psicológico, nutricional y kinésico, constituyen la única terapia efectiva; pues por una parte, alrededor de un $40 \%$ de los pacientes sufren de enfermedades asociadas (hipertensión arterial, diabetes mellitus, dislipidemias, artropatías, trastornos psiquiátricos, respiratorios, enfermedad por reflujo gastroesofágico, etc.) (Erens \& Primatesta, 1999), y porque requieren de un enfoque interdisciplinario que satisfaga las necesidades de atención de los múltiples problemas que pueden coexistir antes y después de la cirugía. No obstante todo esto, los costo involucrados en tratamientos médicos infructuosos, incluidos los relativos a comorbilidad; superan ampliamente los costos de una cirugía electiva debidamente indicada y planificada.

Desde el inicio de la cirugía de la obesidad, hace más de 50 años, se ha intentado optimizar los resultados

* Departamento de Cirugía y Centro de Excelencia en Estudios Morfológicos y Quirúrgicos (CEMyQ), Universidad de La Frontera, Temuco, Chile.

** Clínica Mayor (Red de Clínicas Regionales), Temuco, Chile.

**** Centro de Investigación en Ciencias Biomédicas, Universidad Autónoma de Chile, Temuco, Chile.

***** Servicio de Cirugía, Hospital El Carmen, Maipú, Santiago, Chile. 
postoperatorios con las diversas técnicas disponibles, pero en términos generales las condiciones que debiera cumplir la operación "ideal" son las siguientes: Que sea de bajo riesgo (mortalidad inferior a 1\% y morbilidad inferior al 10\%); que con ella se pueda obtener una disminución cercana al 50\% de pérdida del exceso de peso (PEP) mantenida por al menos 5 años, y que beneficie a más del $75 \%$ de los pacientes operados; que permita una buena calidad de vida, con pocos efectos secundarios; que tenga un bajo índice de reoperaciones (inferior a un $2 \%$ al año); que pueda ser reproducible, con una curva de aprendizaje accesible (Baltasar et al., 2001).

En términos generales, se acepta universalmente como indicaciones para cirugía en obesidad pacientes de entre $15 \mathrm{y}$ 65 años con IMC > 40; IMC > 35 con co-morbilidad coexistente; sujetos con 45 kilos o $100 \%$ por arriba del peso deseado; y, al fracaso de terapias no quirúrgicas para reducir el peso (Sauerland et al., 2005; Ballantyne, 2000).

Este manuscrito ha sido redactado siguiendo la lista de chequeo para estudios observacionales descriptivos (Manterola \& Astudillo, 2013).

El objetivo de este estudio, fue reportar los resultados de una serie de pacientes intervenidos quirúrgicamente por OM con gastrectomía vertical y by-pass de yeyuno (GVBY), en términos de reducción del IMC.

\section{MATERIAL Y MÉTODO}

Se diseñó un estudio de serie de casos prospectiva con seguimiento. Se incluyeron pacientes intervenidos quirúrgicamente por $\mathrm{OM}$ con y sin comorbilidad asociada, en el Servicio de Cirugía del Hospital Clínico de la Universidad Mayor de la ciudad de Temuco, Chile; en el período marzo de 2009 y marzo de 2014, por el primer autor. No se aplicaron criterios de exclusión.

Como protocolo de estudio, se confeccionó una hoja de recogida de datos en las que se registraron las características biodemográficas y clínicas de los pacientes, los hallazgos de laboratorio e imágenes; y la evolución postoperatoria en términos de MPO y otras variables relacionadas con el seguimiento (evolución de IMC, PEP y comorbilidades encontradas en el estudio preoperatorio).

Entre las variables de interés, la variable resultado principal fue reducción del IMC. Otras variables de interés fueron tiempo quirúrgico, estancia hospitalaria, desarrollo de MPO (la que se trabajó de forma dicotómica [no/sí], y por gravedad, aplicando la escala de Dindo y Clavien (Clavien et al., 2009) para lo que se exigió un seguimiento mínimo de 30 días); necesidad de reintervenciones, mortalidad, tiempo de seguimiento; reducción de peso a los 3,6 , $9,12,18,24$ y 36 meses; reducción de la co-morbilidad, porcentaje de pérdida del exceso de peso (\%PEP); porcentaje de éxito y porcentaje de fallo del procedimiento,

Como protocolo de seguimiento, se realizó control clínico y de laboratorio general al $7^{\circ}$ día (curación y retiro de puntos); y luego en los meses 1, 6 y 12; y de ahí en adelante una vez por año del postoperatorio.

Se realizó una GVBY en todos los casos, según técnica previamente descrita por Alamo et al. (2012); que consiste en:

Gastrectomía vertical. Se realizó sello y sección de los vasos cortos con LigaSureTM Vessel Sealing (Covidien Surgical Solutions); y disección del omento mayor $3 \mathrm{~cm}$ proximal al píloro para realizar el primer disparo de sutura mecánica lineal de carga verde (Endo GIATM de $60 \mathrm{~mm}$, Covidien Stapling Solutions). Posteriormente, otros disparos con cargas violetas (Endo GIATM de $60 \mathrm{~mm}$, Covidien Stapling Solutions), son utilizados para dividir longitudinalmente el estómago, desde el antro hasta el ángulo de His. En el intertanto, el anestesiólogo insertó una sonda de calibración orogástrica de 34-French para guiar la sección gástrica. Una vez concluida esta, se realizó una sutura invaginante de la línea de grapas con monofilamento absorbible Monosyn ${ }^{\circledR}-000$ (B. Braun, Sharing Expertise). Posteriormente, y en pacientes seleccionados, se realizó una prueba con azul de metileno a través de la sonda de calibración, para comprobar la indemnidad y ausencia de fugas de la línea de sutura gástrica. Una vez finalizado este paso, se disecó el resto del omento mayor, cerca de la curva gástrica, extirpando posteriormente el estómago seccionado; y retirando la sonda gástrica de calibración.

By-pass de yeyuno. Se identificó el ángulo duodenoyeyunal (de Treitz) y se contaron 20 a $40 \mathrm{~cm}$ a partir de este punto (dependiendo de la movilidad del mesenterio), para confeccionar una Y-Roux, con una carga de sutura blanca (Endo GIATM de $60 \mathrm{~mm}$, Covidien Stapling Solutions). Posteriormente, se realizó una enteroenterostomía (anastomosis yeyuno-ileal) a unos $300 \mathrm{~cm}$ distales a la sección yeyunal, utilizando el mismo tipo de carga de sutura mecánica, asegurando al menos $200 \mathrm{~cm}$ de intestino absorbente. El defecto intestinal posterior al disparo de sutura mecánica fue reparado con un punto continuo de sutura monofilamento absorbible de 3-0 Monosyn ${ }^{\circledR}-000$ (B. Braun, Sharing Expertise). Finalmente, se realizó el cierre de la brecha mesentérica con el mismo material (Fig. 1). 


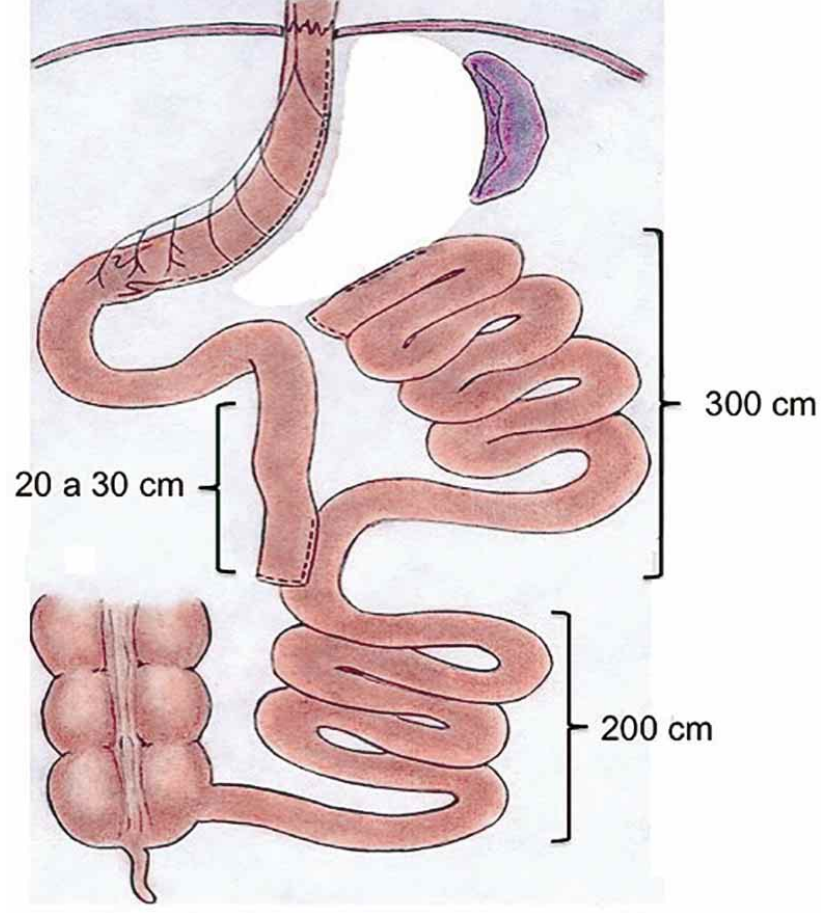

Fig. 1. GVBY. Se construye un tubo gástrico paralelo a la curvatura menor el estómago, desde el área prepilórica de la curvatura mayor hasta el ángulo de His. Posteriormente, se realiza una anastomosis yeyunoileal a aproximadamente 300 cm distal al ángulo duodenoyeyunal (de Treitz).

En casos seleccionados se instaló un drenaje tubular en la cercanía de la línea de sutura gástrica que se exteriorizo por contrabertura y se realizó la rafia de forma convencional.

\section{Definiciones.}

- El exceso de peso en el preoperatorio, se calculó aplicando la fórmula de Hamwi para mujeres ([estatura en centíme- tros - 152,4]/2,54) *2,27+45,4; y para hombres: ([estatura en centímetros $-152,4] / 2,54) * 2,7+48,124$. A partir de esta estimación se calculó el exceso de peso preoperatorio (peso actual - peso ideal).

- La reducción de peso a los 3, 6, 9, 12, 18, 24 y 36 meses se calculó con la siguientes fórmula: (peso inicial - peso postoperatorio a los 3, 6, 9, 12, 18, 24 ó 36 / peso inicial x 100).

- La reducción de la co-morbilidad, se calculó utilizando la siguiente fórmula: (co-morbilidad inicial - co-morbilidad postoperatoria / co-morbilidad inicial x 100).

- El \%PEP se determinó utilizando la siguiente fórmula: (pérdida peso x 100) / (peso inicial - peso ideal). (Manterola, et al., 2003; Manterola, et al. 2005).

Plan de análisis. Se realizó un análisis exploratorio de los datos; posteriormente, se utilizó estadística descriptiva con cálculo de porcentajes, medidas de tendencia central y dispersión (promedios y desviación estándar; mediana y valores extremos).

\section{RESULTADOS}

En el período estudiado, se intervinieron con esta técnica un total de 30 pacientes, con una mediana de edad de 38 años; con un 73,3\% de sexo femenino. Las variables clínicas preoperatorias se describen en las Tablas I y II.

La MPO fue de $13,3 \%$. Se trata de dos casos de fuga anastomótica de la GV y de hemorragia digestiva alta (HDA) en la anastomosis yeyuno ileal. Los casos de fuga anastomótica requirieron de reintervención quirúrgica (índice de reintervenciones de 6,7\%); y los de HDA, sólo medidas generales y terapia ferrosa en uno de ellos. Esta variable se detalla en la Tabla III.

Tabla I. Distribución de variables continuas preoperatorias. $(n=30)$.

\begin{tabular}{lccc}
\hline Variable & Promedio \pm DE & Mediana & Valores extremos \\
\hline Edad (años) & $36,3 \pm 10,8$ & 38 & $18-68$ \\
Est atura (m) & $1,63 \pm 0,08$ & 1,67 & $1,50-1,80$ \\
Peso (Kg) & $106,7 \pm 21,5$ & 121,5 & $70,5-149$ \\
IMC (kg/m2) & $40,1 \pm 6,7$ & 42,8 & $28,3-51,0$ \\
Exceso de peso (Kg) & $50,3 \pm 17,7$ & 59,4 & $20,1-83,8$ \\
Tiempo quirúrgico (min) & $134,6 \pm 22,2$ & 125 & $95-190$ \\
Estancia hospitalaria (días) & $5,3 \pm 7,1$ & 3 & $3-36$ \\
Seguimiento (meses) & $22,9 \pm 11,9$ & 25,5 & $3-40$ \\
\hline
\end{tabular}

$\mathrm{DE}=$ Desviación estándar. 
Tabla II. Distribución de variables categóricas preoperatorias $(\mathrm{n}=30)$.

\begin{tabular}{lcc}
\hline Variable & n casos & \% \\
\hline Sexo & 22 & 73,3 \\
Femenino & 8 & 26,7 \\
$\quad$ Masculino & & \\
Comorbilidad $(*)$ & 0 & 0,0 \\
$\quad$ Sin comorbilidad & 19 & 63,3 \\
Esteatosis hepática & 16 & 53,3 \\
Resistencia insulina & 14 & 46,7 \\
Dislipidemia & 9 & 30,0 \\
HTA & 9 & 30,0 \\
DM $(* *)$ & 8 & 26,7 \\
ERGE $(* *)$ & 8 & 26,7 \\
Colelitiasis & 6 & 20,0 \\
Patología osteomuscular & 6 & 20,0 \\
Depresión & 4 & 13,3 \\
Apnea del sueño & 2 & 6,7 \\
Infertilidad & 2 & 6,7 \\
Hiperuricemia &
\end{tabular}

$(*)=$ Todos pacientes tienen más de una comorbilidad; $(* *)=$ Una paciente DM insulino requirente; $(* * *)=$ Enfermedad por reflujo gastroesofágico.

El seguimiento permitió verificar una reducción progresiva y sostenida del peso, del IMC (normalizado en promedio a los 12 meses, Fig. 2), de la reducción de peso y del \%PEP a lo largo del tiempo, en sus distintas mediciones (Tabla IV).

Tabla III. Morbilidad verificada en los pacientes en estudio $(n=30)$.

\begin{tabular}{lcc}
\hline Variable & n casos & \% \\
\hline Clavien & & \\
Grado 0 & 22 & 86,7 \\
Grado II & 2 & 6,7 \\
Grado IIIb & 2 & 6,7 \\
Morbilidad & & \\
No & 27 & 86,7 \\
HDA & 2 & 6,7 \\
Fuga anastomótica & 2 & 6,7 \\
\hline HDA= Hemorragia digestiva alta. & &
\end{tabular}

La reducción general de co-morbilidad fue de $100 \%$ y la resolución total de la comorbilidad general fue de 91,1\% (82/90). La resolución de resistencia a la insulina, dislipidemia, hiperuricemia, DM y apnea del sueño en el $100 \%$ de los pacientes. La resolución de esteatosis hepática fue de 94,7\% (18/19), de HTA de 88,9\% (8/9), de ERGE de 87,5\% (7/8), de patología osteomuscular de 83,3\% (5/6), de depresión de 33,3\% (2/6), y de infertilidad de $50 \%$ (1/2). En los casos en que no se logró resolución de la comorbilidad, se verificó una reducción de ésta, en magnitudes significativas. El comportamiento de este constructo se puede observar en la Fig. 3.

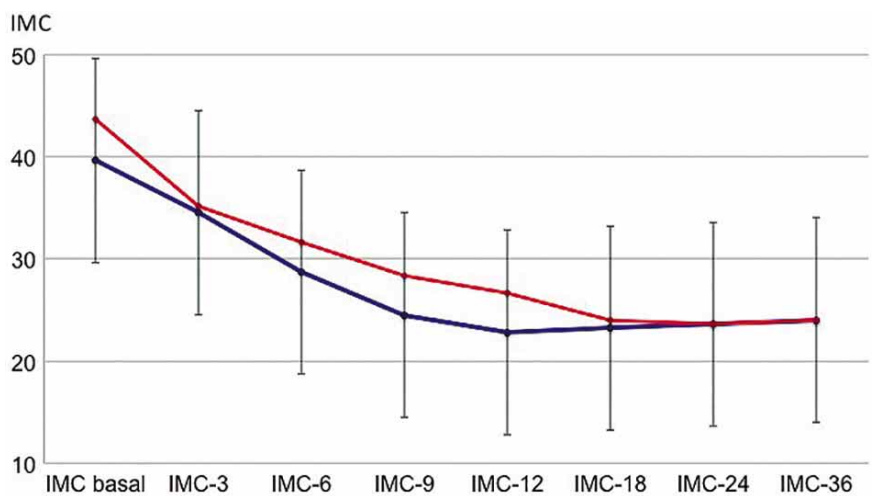

Fig. 2. Gráfico que permite observar el comportamiento de la variable IMC a lo largo del período de seguimiento de la serie, comenzando con la medición basal. La línea azul representa el promedio y la línea roja la mediana de las mediciones de IMC a lo largo del período de seguimiento.

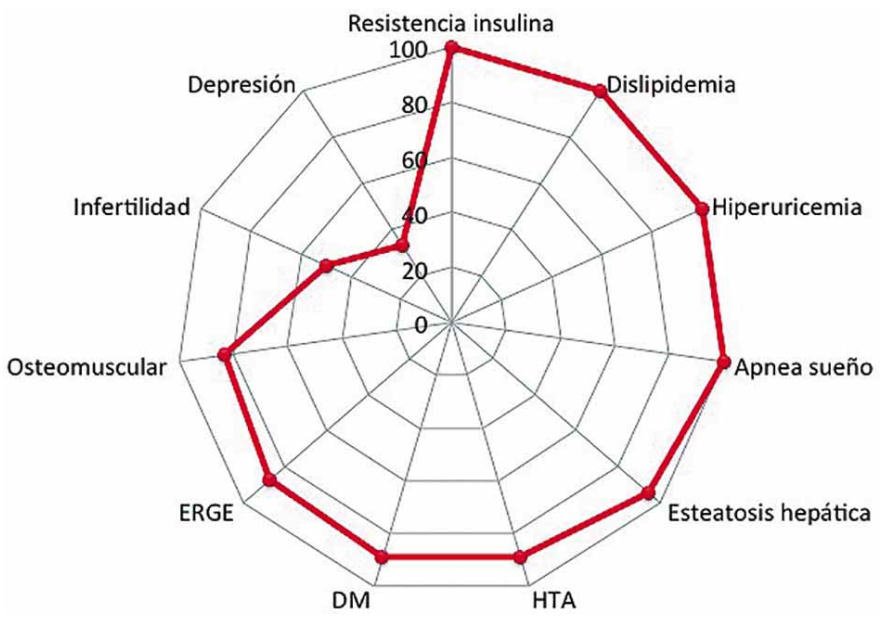

Fig. 3. Gráfico polar o radial, que permite observar el comportamiento de la variable reducción de la comorbilidad, entendiendo esta como un constructo o miniteoría. Se puede apreciar el comportamiento de la reducción de la comorbilidad desde $0 \%$ a $100 \%$ de cada uno de los ítems medidos (DM, HTA, resistencia a la insulina, hiperuricemia, etc.).

Tabla IV. Comportamiento de variables del seguimiento de los pacientes en estudio $(n=30)$.

\begin{tabular}{|c|c|c|c|c|c|c|c|}
\hline \multirow{2}{*}{ Variable } & Preop. & Mes 3 & Mes 6 & Mes 9 & Mes 12 & Mes 18 & Mes 24 \\
\hline & Media $\pm D E$ & Media $\pm D E$ & Media $\pm D E$ & Media+DE & Media $\pm D E$ & Media $\pm D E$ & Media $\pm D E$ \\
\hline Peso $(\mathrm{Kg})$ & $106,7 \pm 21,5$ & $82,5 \pm 15,3$ & $73,2 \pm 13,0$ & $68,6 \pm 10,9$ & $66,4 \pm 9,3$ & $64,1 \pm 10,0$ & $65,0 \pm 12,2$ \\
\hline $\operatorname{IMC}\left(\mathrm{kg} / \mathrm{m}^{2}\right)$ & $39,7 \pm 6,9$ & $31,3 \pm 5,3$ & $28,0 \pm 4,4$ & $26,2 \pm 3,6$ & $25,3 \pm 3,0$ & $24,6 \pm 2,9$ & $24,5 \pm 3,2$ \\
\hline Reducción peso (\%) & --- & $21,1 \pm 5,2$ & $29,9 \pm 5,4$ & $34,1 \pm 6,3$ & $36,6 \pm 8,1$ & $34,6 \pm 8,0$ & $38,0 \pm 6,1$ \\
\hline PEP $(\%)$ & --- & $34,6 \pm 7,6$ & $48,4 \pm 7,8$ & $55,3 \pm 10,5$ & $59,8 \pm 13,5$ & $55,2 \pm 14,5$ & $62,1 \pm 12,4$ \\
\hline
\end{tabular}

$\mathrm{DE}=$ Desviación estándar. 
El resultado de la cirugía en términos de control del peso y de la comorbilidad fue de $100 \%$. Hasta el seguimiento reportado no se verificó fallo del procedimiento.

El comportamiento de otras variables de interés como tiempo quirúrgico, estancia hospitalaria y seguimiento, se detallan en la Tabla I. La serie no reportó mortalidad.

\section{DISCUSIÓN}

La cirugía bariátrica empezó a desarrollarse como técnica para perder peso en 1954 (Greenway, 1996). Posteriormente se fueron clasificando en técnicas restrictivas, mal absortivas y mixtas (Manterola et al., 2005).

El objetivo de la cirugía bariátrica es la pérdida y el mantenimiento de al menos el $50 \%$ del exceso de peso (Balsiger et al., 2000). Sin embargo, más importante que el número absoluto de kilogramos perdidos es la mejoría de las comorbilidades asociadas al sobrepeso. En 1991 el consenso del NIH comunicó que las pérdidas de peso se estabilizan, en general, a los 18-24 meses de la cirugía. No obstante ello, es frecuente la recuperación de peso entre los 2-5 años, pudiendo llegar al fracaso de la técnica (NIH, 1992).

La aparición de complicaciones depende del tipo de intervención, la experiencia del equipo quirúrgico y algunos factores de riesgo como sexo masculino, mayor grado de obesidad coexistencia de comorbilidades como la DM y la HTA. Globalmente se acepta una MPO de hasta un 20\%; destacando la hemorragia digestiva ( $1 \%$ a $4 \%)$, la fuga anastomótica (0\% a 6\%), la infección y la embolia pulmonar (Moreno Esteban \& Zugasti Murillo, 2004; WGO, 2011). Respecto de la MPO observada, vale la pena comentar los casos de fístula gástrica y de HDA de la entero-entero anastomosis (dos casos de cada cual).

En un reporte nacional que compila los resultados de 10 centros chilenos, con un total de 4040 pacientes operados con by-pass gástrico, se describe que la MPO general fue de 2,3 a $20 \%$, con una tasa de reoperaciones de $1,6 \%$ a 11,6\% (Csendes \& Maluenda, 2006).

Respecto de la mortalidad de la cirugía bariátrica, existe evidencia que fluctúa entre $0,05 \%$ y $2 \%$ (que podría considerarse bajo si se toma en cuenta que se trata de cirugía compleja) (WGO).

Por otra parte, es importante recalcar que los pacientes operados con esta técnica no hacen problemas carenciales en el postoperatorio alejado. De todos modos, se suplementó a los pacientes con Centrum ${ }^{\circledR}$ hasta que alcanzaron las 1200 cal de ingesta diaria. Sólo en dos casos observamos alopecia que se resolvió con la administración de zinc. Todos fueron tratados con un plan nutricional progresivo de líquido por pocos días, incrementado progresivamente a papillas y picados según tolerancia (fraccionado cada 2 ó 3 horas) por 4 a 6 semanas; priorizando la ingesta de proteínas de alto valor biológico.

La reducción del exceso de peso y del IMC se logró en todos los pacientes, quienes normalizaron su peso e IMC a los 12 meses, lo que no ocurre con el by-pass gástrico, la manga gástrica ni el by-pass biliopancreático; técnicas con las que después de 24 meses de seguimiento no se logra normalizar estas variables en la totalidad de los pacientes (Ocón Bretón et al., 2005).

Hay evidencia que sustenta que la aplicación de gastrectomía vertical aislada, se asocia a una \%PEP de $61 \%$ (Rosen et al., 2009).

La GVBY se define como una técnica restrictiva (adaptativa) y hormonal, que comenzó a utilizarse en 2004, con el objetivo de preservar la función pilórica y duodenal (Alamo Alamo et al., 2006; de Menezes Ettinger et al., 2006) de tal modo que la exposición temprana de los alimentos no digeridos en el íleon, genere secreción de hormonas incretinas, especialmente GLP-1; la que es producida por las células L del íleon estimulando la secreción de insulina por parte de las células beta (efecto incretina). De este modo, el GLP-1 aumenta la producción de células beta, reduce la apoptosis de éstas; y la secreción de glucagón por parte de las células alpha (Neumiller, 2003); y en conjunto con el péptido YY (PYY), inducen saciedad y vaciamiento gástrico retardado; efecto conocido como "ileal break" o freno ileal (Miller et al., 1981; Layer et al., 1995). Por otra parte, existe evidencia que sustenta que la acción de GLP-1 estimula al centro de la saciedad en el tronco encefálico, enlentece el vaciamiento gástrico; y tendría acciones adicionales como cardioprotector.

El efecto del GLP-1 y de la incretina es también el mecanismo responsable de la mejoría o remisión precoz de la resistencia a la insulina y la DM tipo 2 en pacientes obesos posterior a la cirugía (Bose et al., 2009; Alamo et al.).

Por otra parte la GVBY ha demostrado asociarse a mínimas complicaciones metabólicas comparada con otras técnicas como el by-pass gástrico y la diversión biliopancreática con switch duodenal (Mechanick et al., 2008) puesto que lo que se persigue con esta técnica no es la 
malabsorción sino la liberación de hormonas. Lo que se verifica, pues no hay pacientes con hipoalbuminemia ni carencias.

No se han medido niveles de micronutrientes ni de vitaminas, razón por la que no podemos estar seguros que no existan deficiencias en el postoperatorio de estos pacientes; sin embargo, los niveles de albumina y linfocitos han sido normales; y no hemos observado casos de diarrea, síndrome de dumping, pérdida excesiva de peso o anemia. Esta última, se aprecia en cifras de $6 \%$ a $50 \%$ en pacientes sometidos a by-pass gástrico (Cable et al., 2011; Alvarez-Cordero \& Aragon-Viruette, 1992; Halverson, 1986; Simon et al., 1989), técnica que también se asocia al desarrollo de dumping (Loss et al., 2009). Por otra parte, tampoco se han evidenciado signos de disfunción hepática.

Ahora bien, el derivar 3 o más metros de intestino delgado, podría asociarse al desarrollo de síndrome de sobrecrecimiento bacteriano o síndrome de asa ciega; sin embargo, el desarrollo de esta entidad, requiere tránsito intestinal alimentario enlentecido como sustrato bacteriano, tal y como ocurre en sujetos con aganglionosis intestinal y pancreatitis crónica (Bures et al., 2010; Quigley \& Abu-
Shanab, 2010). En la GVBY el segmento intestinal derivado no tiene tránsito alimentario, pero si isoperistalsis y producción mucosa de IgA.

Este estudio tiene algunas limitaciones evidentes que ameritan ser comentadas: una se refiere a la heterogeneidad de la población estudiada (se reportan todos los pacientes que han sido tratados de esta forma en el HCUM durante el período de tiempo señalado); otra es lo heterogéneo del seguimiento de estos; y otra, es que se trata de un pequeño número de casos.

A modo de conclusión, podemos señalar que los resultados observados con GVBY son comparables con los de otras series de cirugía de OM.

\section{AGRADECIMIENTOS}

Agradecemos la colaboración de Covidien Surgical Solutions; B. Braun, Sharing Expertise; y Aesculap Surgical Instruments; quienes nos han dado soporte para el desarrollo de esta iniciativa.

MANTEROLA, C.; ALAMO, M.; HORTA, J.; ICARTE, M. A.; RIVEROS, C.; AYALA, C. \& MENDOZA, E. Initial results of weight loss surgery with vertical gastrectomy and jejunal bypass. Int. J. Morphol., 32(3):991-997, 2014.

SUMMARY: Morbid obesity (MO) is a chronic disease that is associated with risk of morbidity and mortality. Surgery has proven to be the best treatment option. The objective of this study is to report the results of a series of patients undergoing surgery for MO with vertical gastrectomy and jejunal bypass (VGJB) in terms of BMI reduction. Prospective case series. All patients undergoing surgery or MO during the 2009 to 2014 period, in the Surgery Department of the Hospital Clinico de la Universidad Mayor, Chile, were included. All patients were operated with a VGJB. The main outcome variable was BMI reduction. Other variables of interest were operative time, hospital stay, postoperative morbidity (POM), excess weight loss (EWL), percentage of excess weight loss (\%EWL), reduction in associated morbidity and mortality. Descriptive statistics were used calculating percentages, measures of central tendency and dispersion. Thirty patients, with a median age of 38 years, 73,3\% female, were operated. A progressive and significative reduction of BMI (normalized at 12 month follow-up) EWLand \%EWL. Reduction in associated morbidity was $100 \%$. Median of operative time and hospital stay were 125 min and 3 days, respectively. POM was $13.3 \%$ (two cases of anastomotic leak and two of upper gastrointestinal bleeding). No mortality was reported in this series of patients. Observed results with VGJB are comparable with other series of surgery for MO.

KEY WORDS: Morbid obesity; Morbid obesity/surgery; Bariatric surgery; Body Mass Index.

\section{REFERENCIAS BIBLIOGRÁFICAS}

Alamo Alamo, M.; Sepúlveda Torres, M. \& Zapata Perez, L. Vertical isolated gastroplasty with gastro-enteral bypass: preliminary results. Obes. Surg., 16(3):353-8, 2006.

Alamo, M.; Sepúlveda, M.; Gellona, J.; Herrera, M.; Astorga, C. \& Manterola, C. Sleeve gastrectomy with jejunal bypass for the treatment of type 2 diabetes mellitus in patients with body mass index $<35 \mathrm{~kg} /$ m2. A cohort study. Obes. Surg., 22(7):1097-103, 2012.

Alvarez-Cordero, R. \& Aragon-Viruette, E. Post-operative Complications in a Series of Gastric Bypass Patients. Obes. Surg., 2(1):87-9, 1992.
Atalah, E. Epidemiología de la obesidad en Chile. Rev. Med. Clin. Condes, 23(2):117-23, 2012.

Ballantyne, G. H. Bariatric surgery: SAGES guidelines for laparoscopic and conventional surgical treatment of morbid obesity. Santa Monica, Society of American Gastrointestinal Endoscopic Surgeons, 2000. Disponible en http://www.lapsurgery.com/SAGES.htm

Balsiger, B. M.; Murr, M. M.; Poggio, J. L. \& Sarr, M. G. Bariatric surgery. Surgery for weight control in patients with morbid obesity. Med. Clin. North Am., 84(2):477-89, 2000. 
Baltasar, A.; Bou, R.; Bengochea, M.; Arlandis, F.; Escrivá, C.; Miró, J.; Martínez, R. \& Pérez, N. Duodenal switch: an effective therapy for morbid obesity--intermediate results. Obes. Surg., 11(1):54-8, 2001.

Bose, M.; Oliván, B.; Teixeira, J.; Pi-Sunyer, F. X. \& Laferrère, B. Do Incretins play a role in the remission of type 2 diabetes after gastric bypass surgery: What are the evidence? Obes. Surg., 19(2):217-29, 2009.

Bures, J.; Cyrany, J.; Kohoutova, D.; Förstl, M.; Rejchrt, S.; Kvetina, J.; Vorisek, V. \& Kopacova, M. Small intestinal bacterial overgrowth syndrome. World J. Gastroenterol., 16(24):2978-90, 2010.

Cable, C. T.; Colbert, C. Y.; Showalter, T.; Ahluwalia, R.; Song, J.; Whitfield, P. \& Rodriguez, J. Prevalence of anemia after Roux-en-Y gastric bypass surgery: what is the right number? Surg. Obes. Relat. Dis., 7(2):134$9,2011$.

Clavien, P. A.; Barkun, J.; de Oliveira, M. L.; Vauthey, J. N.; Dindo, D.; Schulick, R. D.; de Santibañes, E.; Pekolj, J.; Slankamenac, K.; Bassi, C.; Graf, R.; Vonlanthen, R.; Padbury, R.; Cameron, J. L. \& Makuuchi, M. The Clavien-Dindo classification of surgical complications: fiveyear experience. Ann. Surg., 250(2):187-96, 2009.

Csendes, A. \& Maluenda, F. Morbimortalidad de la cirugía bariátrica. Experiencia chilena en 10 instituciones de salud. Rev. Chil. Cir., 58(3):208$12,2006$.

de Menezes Ettinger, J. E.; Azaro, E.; Mello, C. A. \& Fahel, E. Analysis of the vertical isolated gastroplasty: a new bariatric operation. Obes. Surg., 16(9):1261-3, 2006.

Erens, B. \& Primatesta, P. Health Survey for England: Cadiovascular disease 1998. London, The Stationery Office, 1999.

Greenway, F. L. Surgery for obesity. Endocrinol. Metab. Clin. North Am., 25(4):1005-27, 1996.

Halverson, J. D. Micronutrient deficiencies after gastric bypass for morbid obesity. Am. Surg., 52(11):594-8, 1986.

Layer, P.; Holst, J. J.; Grandt, D. \& Goebell, H. Ileal release of glucagonlike peptide-1 (GLP-1). Association with inhibition of gastric acid secretion in humans. Dig. Dis. Sci., 40(5):1074-82, 1995.

Loss, A. B.; de Souza, A. A.; Pitombo, C. A.; Milcent, M. \& Madureira, F. A. Analysis of the dumping syndrome on morbid obese patients submitted to Roux en Y gastric bypass. Rev. Col. Bras. Cir., 36(5):4139, 2009.

Manterola D., C.; Pineda N., V.; Vial G., M.; Losada M., H. \& Muñoz N. S. Cirugía bariátrica. Elección de un tipo de técnica basado en la evidencia. Revisión sistemática de la literatura. Rev. Chil. Cir., 55(4):398404, 2003

Manterola, C.; Pineda, V.; Vial, M.; Losada, H. \& Muñoz, S. Surgery for morbid obesity: selection of operation based on evidence from literature review. Obes. Surg., 15(1):106-13, 2005.

Manterola, C. \& Astudillo, P. Checklist for reporting of descriptive observational studies. MINCIR Initiative. Int. J. Morphol., 31(1):115$20,2013$.

Mechanick, J. I.; Kushner, R. F.; Sugerman, H. J.; Gonzalez-Campoy, J. M.; Collazo-Clavell, M. L.; Guven, S.; Spitz, A. F.; Apovian, C. M.; Livingston, E. H.; Brolin, R.; Sarwer, D. B.; Anderson, W. A. \& Dixon,
J. American Association of Clinical Endocrinologists, The Obesity Society, and American Society for Metabolic \& Bariatric Surgery Medical guidelines for clinical practice for the perioperative nutritional, metabolic, and nonsurgical support of the bariatric surgery patient. Endocr. Pract., 14 Suppl. 1:1-83, 2008.

Miller, L. J.; Malagelada, J. R.; Taylor, W. F. \& Go, V. L. Intestinal control of human postprandial gastric function: the role of components of jejunoileal chyme in regulating gastric secretion and gastric emptying. Gastroenterology, 80(4):763-9, 1981.

Moreno Esteban, B. \& Zugasti Murillo, A. Bariatric surgery: an update. Rev. Med. Univ. Navarra, 48(2):66-71, 2004.

National Institutes of Health (NIH). Gastrointestinal surgery for severe obesity: National Institutes of Health Consensus Development Conference Statement. Am. J. Clin. Nutr., 55(2 Suppl.):615S-619S, 1992.

Neumiller, J. J. Differential chemistry (structure), mechanism of action, and pharmacology of GLP-1 receptor agonists and DPP-4 inhibitors. J. Am. Pharm. Assoc., 49 Suppl. 1:S16-29, 2009.

Ocón Bretón, J.; Pérez Naranjo, S.; Gimeno Laborda, S.; Benito Ruesca, P. \& García Hernández, R. Eficacia y complicaciones de la cirugía bariátrica en el tratamiento de la obesidad mórbida. Nutr. Hosp., 20(6):409-14, 2005.

Quigley, E. M. \& Abu-Shanab, A. Small intestinal bacterial overgrowth Infect. Dis. Clin. North Am., 24(4):943-59, 2010.

Rosen, D. J.; Dakin, G. F. \& Pomp, A. Sleeve gastrectomy. Minerva Chir., 64(3):285-95, 2009

Sauerland, S.; Angrisani, L.; Belachew, M.; Chevallier, J. M.; Favretti, F.; Finer, N.; Fingerhut, A.; Garcia Caballero, M.; Guisado Macias, J. A.; Mittermair, R.; Morino, M.; Msika, S.; Rubino, F.; Tacchino, R.; Weiner, R. \& Neugebauer, E. A. M. Surg. Endosc., 19(2):200-21, 2005.

Simon, S. R.; Zemel, R.; Betancourt, S. \& Zidar, B. L. Hematologic complications of gastric bypass for morbid obesity. South. Med. J., 82(9):1108-10, 1989.

World Gastroenterology Organization (WGO). Guías Mundiales de la Organización Mundial de Gastroenterología. Obesidad. 2011. Disponible en: http://www.worldgastroenterology.org/assets/export/userfiles/ Obesity_MASTER_SP.pdf

Dirección para Correspondencia:

Dr. Carlos Manterola

CEMyQ

Universidad de La Frontera

Temuco

CHILE

Email: carlos.manterola@ufrontera.cl 